\title{
Educational Tool for Nanophysics Using Multisensory Rendering
}

\author{
F. Marchi ${ }^{1,2}$, D. Urma ${ }^{1}$, S. Marliere ${ }^{1}$, J.L. Florens ${ }^{1}$, A. Besancon ${ }^{3}$, J. Chevrier ${ }^{2}$, A. Luciani ${ }^{1}$ \\ ${ }^{1}$ ICA-ACROE, 46 Av. Felix Viallet, 38031 Grenoble Cedex, France \\ ${ }^{2}$ LEPES, CNRS, 25 Avenue des Martyrs, 38042 Grenoble Cedex 9, France \\ ${ }^{3} L A G, I N P G, B P$ 46, 38402 Saint Martin d'Heres, France \\ E-mail: marchi@grenoble.cnrs.fr
}

\begin{abstract}
The presentation and the evaluation of an educational tool used to teach physical phenomena that take place at a nanometer scale are the central objectives of the work presented in this paper. Through concepts and tools, the real phenomena are connected to the virtual world, which furnish sensorial representations (haptic, visual, auditory) for the student who is experimenting.

A computer representation of the nanoscene at the atomic level offers a multisensory tangible artifact of nanoworld inaccessible entities. The parameters accessibility allows students to design by themselves and perceive various contact interactions, to understand the origins of complex phenomena as the approach-retract nano-palpating effect.

An interactive way for teaching and understanding the differences between the nanoscale physics and the macroscale physics is illustrated through an experience when students shake the Drosophilae leg.

Real nanomanipulations on various samples by means of the developed multisensorial platform confirm better results in student comprehension and dexterity for achieving the same item as with the classical tools.
\end{abstract}

\section{Introduction}

Nanophysics has opened a broad spectrum in human knowledge and understanding of the nontangible phenomena but also a variety of new questions. How to adapt teaching of science and to reconcile human perception with mathematical and scientific views of reality is one of these questions.

In the nanoworld, the dominant physical phenomena are not anymore the gravity. Adhesion and friction phenomena [1] are much more relevant and they eventually suppress inertial motion. In order to manipulate nanobjects fixed on surfaces we have to compensate adhesive forces with different origins as capillarity, Van der Waals, electrostatic attraction depending on the environment. Due to these specific behaviours, the strategies and tools used in the nanoworlds to move and interact with an object cannot be simply transferred from the macroscopic scale. Today, atomic force microscopy is one of the most widespread techniques to characterize surfaces from micro to atomic scale and also a powerful tool to manipulate nano-objects. Nevertheless, the conventional interface of these microscopes is not an interactive one, does not offer real-time information, collected data being only visually represented as topographic images or curves. Interpretation of this data is not an intuitive task and requires time and solid theoretical background.

New methods of teaching and experimenting nanophysics are needed to make understandable the nanoworld phenomena to a large number of students. In order to underline the principle of nanoscale interactions we have linked a multisensorial platform to an Atomic Force Microscope (AFM). Thus, a student is immerged in the nanoscene through a realtime modelling station equipped with a force feedback device and generating the visual and auditory scene in accordance to the dynamics of the phenomena. He/she is present in the interaction since he/she can act on scene and perceives the nano-scale effects through the main sensorial channels: haptic, vision, audition.

In this paper we will describe the interactive multisensorial platform we have developed in educational aims. Firstly, some previous experiences using haptic interfaces in learning science are presented and followed by the description of the physical phenomenon, which has to be understood by students during our practical works.

Secondly, the augmented reality system with its components, functionalities and characteristics asked by the pedagogical application is described, as well as the principle of physically based modelling engine. 
The role that mixed reality plays in student training is illustrated through the implemented models.

Finally, results and discussions on the efficiency and the necessity of adding multisensory feedback in addition to a commercial AFM, especially in educational purposes are provided through the practical works done by students in Physics at Master level and an educational project on non-typical samples.

\section{Background}

\subsection{Using force feedback in learning science}

Some previous educational approaches have used virtual reality applications with haptic, visual, and/or auditory feedback in different ways to improve scientific learning in biology [2] or chemistry [3]. For example, in the aim of teaching atomic bonding, a research group from Baltimore [4] has coupled a force feedback system to own-developed molecular modelling software. Their work has shown a significant improvement in the time required to create a simple molecule and to understand bonding rules by the use of a force feedback device.

In nanophysics education, Murphy [5] has implemented a method of using atomic and magnetic force microscopy to led the students in differentiating ranges and origin of forces involved in imaging, while Ringlein [6] has developed simulations to describe and illustrate the atomic origins of friction. Their works even if students oriented are based especially on visual rendering and does not include full immersion of the student at the nanometer scale.

For research use, some groups have combined an Atomic Force Microscope (AFM) with haptic devices and virtual reality interface to facilitate nanomanipulation $[7,8]$. On the same purpose, $\mathrm{Li}$ and colleagues [9] have improved the on-line observation of the changes in nano-environment by developing the real-time visual display combined with the real-time force feedback. All these studies improve the AFM capabilities, which help the operator to perform nanomanipulations but do not facilitate the nano-scene phenomena understanding and the control of tipsample interactions.

A better understanding of a nanoscene phenomenon is possible only by seeing, hearing and, the most important, feeling simultaneously the dynamics of the nanoscene. This paper focuses on the approach-retract phenomenon that exists in most of the nano-object manipulations by an AFM probe. The main goal of the work is to render this complex phenomenon as intuitive to understand as possible. For that, visual and auditory metaphors are implemented. A real-time modelling engine generates realistic virtual nano-scenes based on physical models. The presence of the modular force feedback device, the visual and auditory scenes leads to an attractive and enjoyable interface for the user. On one hand, the coupling of the virtual models to the sensorial interface immerse students in a virtual nano-world and on the other hand, the coupling of this multisensorial platform with the AFM allows the students to interact directly with the real nano-world. These two different coupling enhance the learning process in comparison to the traditional approaches based mainly on curve analysis. The results of our approach have been evaluated from the comparison between the quality of the practical work reports written by students who have used multisensorial interface and the one produced by students who have used classical AFM interface.

\subsection{Approach-retract phenomenon}

When two atoms approach to each other in vacuum, they start interacting and form a bond stabilised at the nanoscale by the Van der Waals interaction. The force given by the derivative of the Lennard-Jones potential represents the intensity of the bonding as a function of the distance, $z$ [10]. For two atoms interaction this force has the following expression:

$$
F=-\operatorname{grad}\left(U_{L J}\right)=\frac{12 B}{z^{13}}-\frac{6 A}{z^{7}}(1)
$$

where $\mathrm{A}$ and $\mathrm{B}$ are characteristic constants of the material.

The curve given by this force is constituted by an attractive branch, Van der Waals interaction, which describes long-range interactions $\left(z^{-7}\right)$ and by a repulsive branch, which becomes dominant for short distances $\left(z^{-13}\right)$. Although the contact interaction between an AFM tip and a nano-surface follows this curve variation, as the tip is linked to the piezo tube through a cantilever, the spring constant, $K$, of the cantilever has to be taken into account as it determines the contact behaviour.

As the tip-surface distance decreases, the successive cantilever deflexions, proportional to the force intensity of the tip apex-surface interaction, are the data provided by an AFM device [11]. The total potential that describes the AFM probe behaviour (piezoelectric tube, cantilever and tip assembly) is given by the equation (2):

$$
U_{\text {total }}=U_{C}+U_{L J} \text { where } U_{C}=K \frac{z^{2}}{2}
$$

Usually, the AFM recorded data in contact mode are force-distance curves, force values corresponding to the derivative of the total potential (Fig. 1).

These curves present two main characteristics: (1) two fast force variations, one in the approach phase called 'snap on' of the tip to the surface and a second one in the retract phase called 'snap out'; (2) a hysteresis behaviour, which impedes two different 
thresholds between the approach phase and the retract phase. These behaviours are determined by the system instabilities.

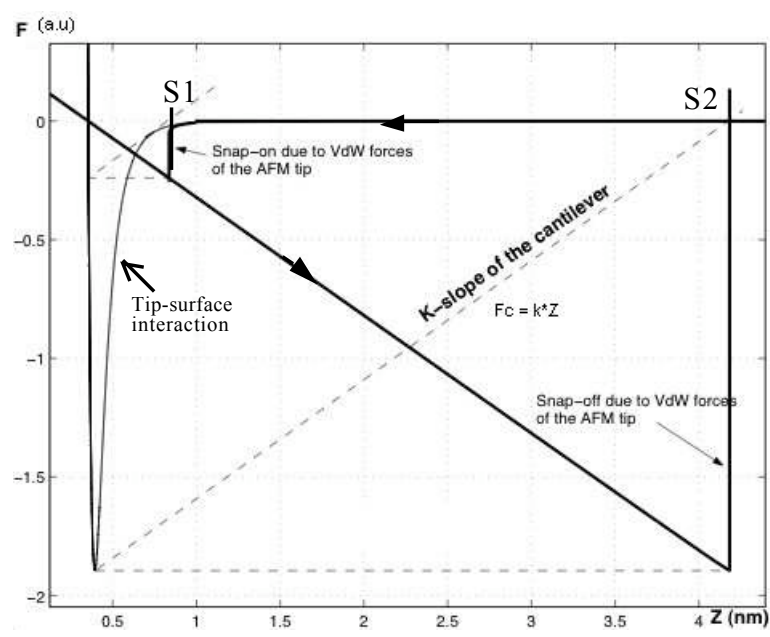

Fig. 1. Force-distant curve for approachretract phenomenon

The physical origin of these two main specificities is then not straightforward to be understood by the students just by analysing a force curve. One idea is to sensorial reconstruct this interaction, and thus, the differences in position thresholds are outlined by the visual rendering, the temporal positioning of the phenomenon is render by the active sound while the differences in force intensities are felt by means of a force feedback device.

\section{Design of the educational platform}

\subsection{Hardware description}

The developed augmented reality system (Fig. 2) brings together (1) performant tools like the Atomic Force Microscope, the Real-Time Modelling Station and the Modular Gestural Device all constituting the physical layer of the instrument; (2) multisensorial representations based on physical modelling forming the energetically coherent and synchronized functional layer and (3) symbolic representation of the phenomena situated in the psycho cognitive layer.

The real-time modelling station placed between the AFM and the force feedback device assures the synchronized data flow $(3 \mathrm{kHz}$ frequency in reactive mode) between the experimentalist and the nanoworld but, the most important, it assures the support for implementing multisensory metaphors defining the active visual and auditory displays. Physical-based models implemented on this real-time computation engine provide the visual and auditory changes according to the dynamics of the manipulated scene. The sonification process is placed in its co-ordination with the haptic and vision sensory processes during action for completing the multi-sensory rendering of the desired phenomena.

In order to offer a suitable environment matching any kind of nanomanipulation, the user should be able to interact with the real and virtual nano-scene through a variable number of degree of freedom (DOF). The implemented haptic display presents a modular interface [12]. Thus, according to the specific manipulation, the experimentalist can use either a key of a piano-keyboard that offers the same up-and-down movements as the AFM tip during an approach-retract interaction or a three-dimensional stick that allows 3DOF movements needed for a nano-object manipulation.

The nanomanipulator viewed as an entity is a complex and new dynamical system. The interaction with the human operator takes place through the force feedback system, which is the key physical element to reconstruct the effects of nano phenomena accessible to the human gesture, and thus to transfer the information

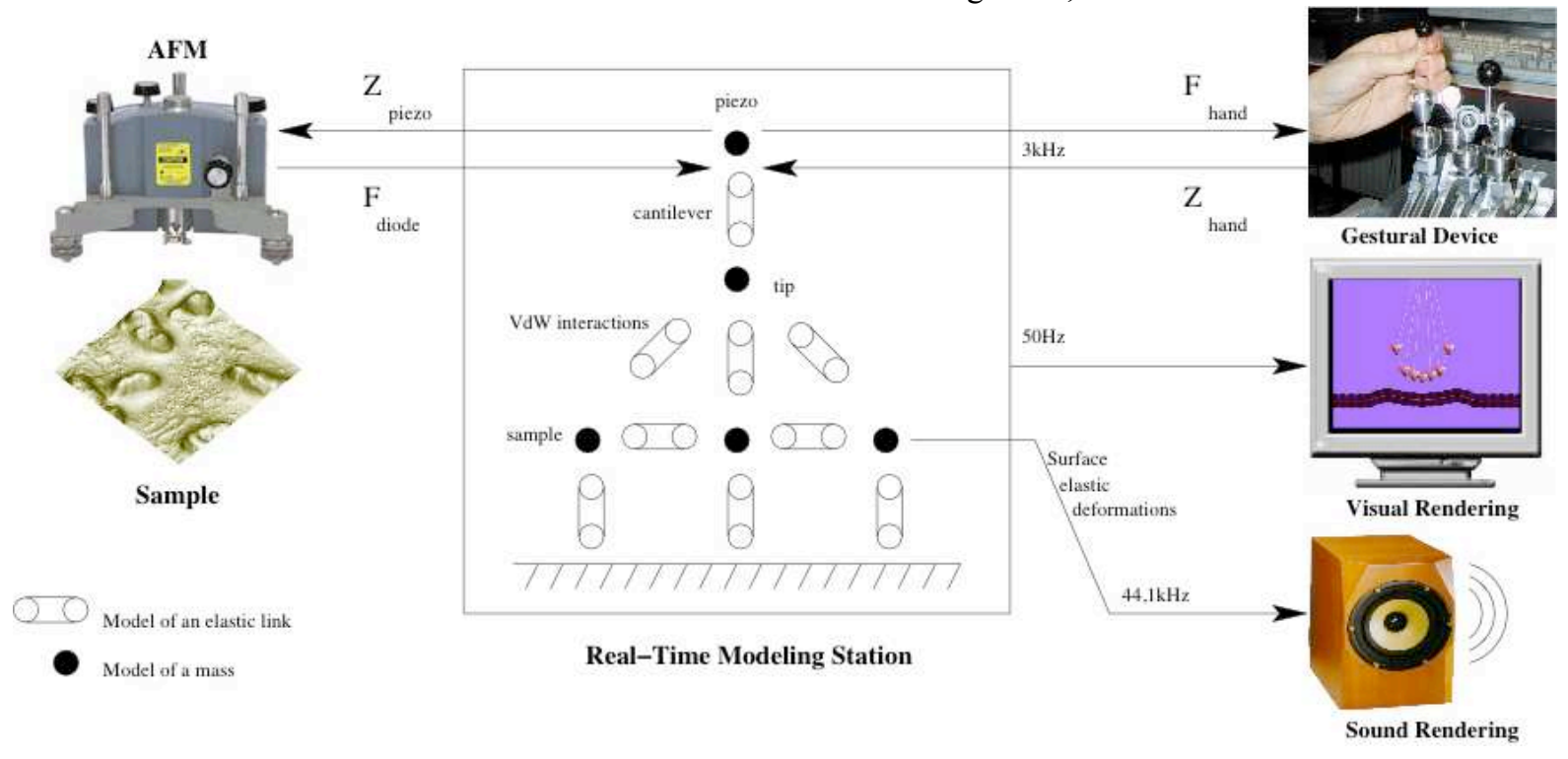

Fig. 2 Augmented reality system 
between nanoscopic and macroscopic levels. As the dynamics of the haptic system mainly influence the nanomanipulator dynamical performances, some minimal criteria have to be satisfied. The quantitative technological requirements of the manipulated system for a pedagogical application are presented in the following.

The signal bandwidth has to be at least $1 \mathrm{kHz}$. This value is necessary to ensure an accurate rendering of the contact with a very stiff object, at a mechanical level of human perception, which has a much higher dynamics than the human sensori-motor loop. In practice, a bandwidth of $1.5 \mathrm{kHz}$ is attained with an appropriate sampling rate in our instrument.

The latency defined as the delay between the produced signal and the feedback signal as the instrument transmits it. The minimum value attainable with a numerical device is one sampling interval. A higher latency would introduce distortions in the physical phenomena rendering. In our case, the latency is $0,33 \mathrm{~ms}$, the duration of one sampling interval.

The scaling factors (position and force scaling factors) between the human operator and the manipulated object are, in our case, as follows:

a) Position scaling factor $\alpha_{P}$ defined as the ideal ratio between a variation in the nanoprobe position $Z_{\text {piezo }}$ and a variation in the measured position at the human operator level $Z_{\text {hand }}$ :

$$
\alpha_{P}=Z_{\text {piezo }} / Z_{\text {hand }}=0,14 \times 10^{-3}
$$

b) Force scaling factor, $\alpha_{f}$, defined as the ideal ratio between a variation of the force feedback to the human operator $F_{\text {hand }}$ and the variation of the interaction force measured by the AFM, $F_{\text {diode }}$ :

$$
\alpha_{f}=F_{\text {hand }} / F_{\text {diode }}=2 \times 10^{8} \text {. }
$$

The dynamical range of the instrument, which is the ratio between the maximum measurable variation of the force in the force feedback device and in the AFM is $158 \mathrm{~dB}$.

The resemblance between the real physical phenomenon and its representation, gives rise to a good comprehension at a conceptual level. This is a qualitative criterion, but very important in such pedagogical applications. The resemblance is improved in our case by the multisensorial rendering (force feedback, visual and sound rendering).

\subsection{Software description}

The real-time modelling engine runs on the fundamental principles of physical-based simulation. It can be distinguished two kinds of physical values for a space element: the intensive values, such as force and the extensive values, such as position. The formalism, called Cordis-Anima [13] is based on two elements or basic points: the $\mathrm{M}$ point, which receives an intensive value and transmits an extensive value; the $\mathrm{L}$ point, which receives an extensive value and transmits an intensive value.

A simulation module is then a set of $\mathrm{M}$ and $\mathrm{L}$ points. These points are linked together according to three main rules:

- only opposite-type points ( $\mathrm{M}$ with L) can be connected;

- an M point could be connected to several L points: this $\mathrm{M}$ point will then receive the sum of the intensive values sent by the $\mathrm{L}$ points ;

- an L point can be connected to a single M point.

From these assumptions, two types of basic modules MAT and LIA have been set up (Fig. 3), from which any mesh structure can be created. The MAT module, representing a pin-point mass, is made of a single $M$ point and its algorithm in discrete time is calculated from the fundamental relation of dynamics:

$$
X_{n}=2 X_{n-1}-X_{n-2}+\frac{1}{M} F_{n-1}
$$

where $F_{n}=f(n \cdot \Delta t)$ is the sum of the forces received by the $M$ point, $X_{n}=x(n \cdot \Delta t)$ is its position, $f, x$ are the corresponding continuous measures and $M=m / \Delta t^{2}$.
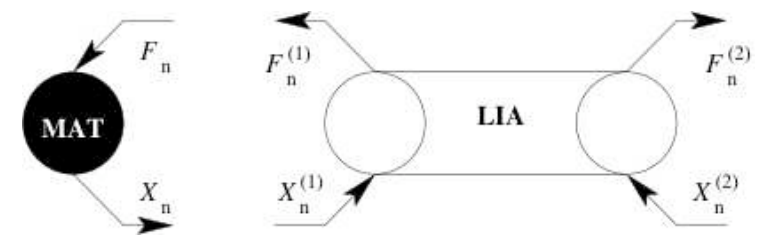

\section{Fig. 3 MAT/LIA link with forces and positions}

The LIA module is composed of two $\mathrm{L}$ points sending two opposite forces, according to actionreaction principle. The elastic link follows the discretetime algorithm:

$$
F_{n}^{(2)}=-F_{n}^{(1)}=-K\left(X_{n}^{(2)}-X_{n}^{(1)}\right)
$$

with $K$ the spring constant.

The modelling algorithm based mainly on two elements (MAT, LIA) allows constructing easily structures from very simple to very complex one. By modifying the parameters of each element: mass, spring constant, viscosity, a wide spectrum of real phenomena can be designed. The algorithm modularity, the facility in building new models in addition to real-time running are satisfied conditions for an educational-adapted tool.

\subsection{Model implementation}

By means of the Cordis-Anima modelling and simulation system, a minimal representation of the real 
and complex phenomenon of approach-retract interaction is implemented. The components involved in this interaction: the piezoelectric tube, the tip and the sample surface defined at an atomic level, are modelled like material objects with their specific mass, that are permanently in interaction with the external environment as well as among them. The equivalent schema is presented in Fig. 4, where the cantilever is represented as a spring with zero length chargeless and driving the tip on the $\mathrm{Oz}$ axis, while the tip-surface interaction is treated as a non-linear atom-atom interaction.

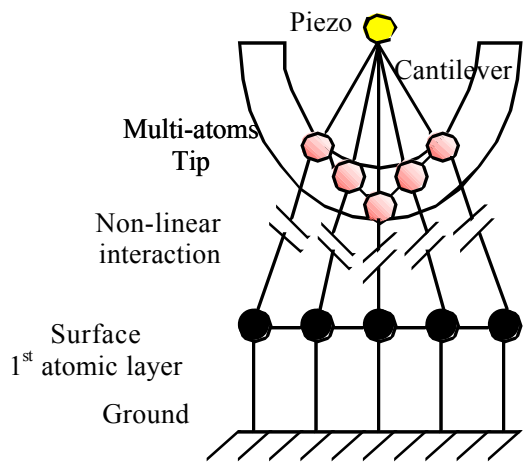

\section{Fig. 4 Model for approach-retract phenomena}

Starting from the equation (1) which typically describes the tip-surface interaction and taking into account the spring constant of the cantilever, $K$, it is possible to find parameters for an interaction linear by pieces that give the same approach-retract curve that the non-linear one. The functional point (Fig. 1) of the system composed by piezoelectric tube, cantilever and tip during the approach-retract phenomenon is the cross-point between the tip-surface characteristic curve and the cantilever characteristic curve (dashed line in Fig.1). Analyzing the AFM functionality from this point of view, an approach-retract interaction curve with its typical hysteresis is obtained and implemented by means of Cordis-Anima modelling.

The results of the virtual nanoscene manipulation are illustrated in Fig. 5. When the approach phenomenon begins, as long as tip-surface distance is great enough, no tip-surface interactions appear (Fig. $5 a)$, so there are no interaction forces. The moment when the distance becomes S1, the "snap-on" threshold from Fig. 1, the AFM tip is attracted by the surface due to Van der Waals forces (Fig. 5b) and the snap of the tip on the surface (Fig. 5c) takes place.

During the retract phenomenon (Fig. 5d), the force necessary to withdraw the tip from the surface has to be stronger than in the approach case. By retracting the piezoelectric tube far apart from the surface (Fig. 5e), the retract threshold S2 from Fig. 1 is reached, the working point suddenly changes, the tip gets back to the piezoelectric tube position and the exerted force get back to zero (Fig. 5f).

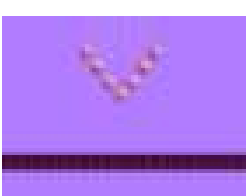

a

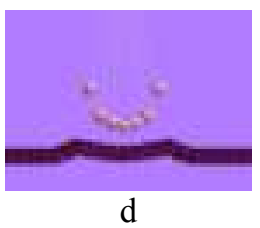

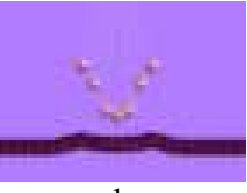

b

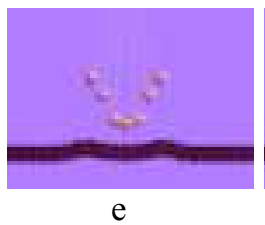

e

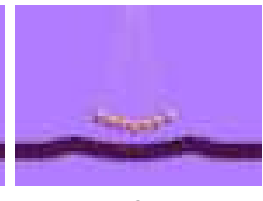

c

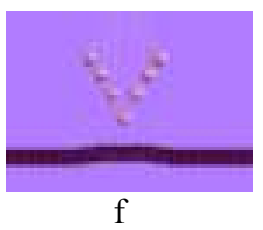

Fig. 5. Representation of the approachretract interaction

The tip-surface interaction implies the energy exchanges between the elements involved in interaction. These exchanges are captured at the atomic layer of the surface (Fig. 4) and used to modulate the sound. Thus, the acoustical vibration of the surface atomic layer is combined with the movement of the symbolic tip and follows the dynamics of the interaction.

By the use of atomic representation as visual metaphor, the real-time sound feedback of the interaction and the force feedback implementation this model allows a better and rapid understanding of the approach-retract phenomenon. A student does not only see and hear how the interaction is propagating but feels also the forces involved in.

\section{Results}

\subsection{Real nano-scene manipulations}

In the aim of teaching physical phenomena nonaccessible by our senses as the cohesive interaction between nano-objects, a three steps methodology is proposed.

Students addressed by this subject have the theoretical background in nano-physics and basic concepts on atomic force and tunnelling microscopy. However, they have no experience in multisensory rendering. A first step of a complete nanomanipulation is their training on virtual models. This will allow not only to understand the complete phenomena, but also to create a multi-sensory environment for testing virtual nanomanipulations. 
Once the student accommodates the functional modes of the real-time modelling station and the use of the force feedback, he/she passes to the real nanomanipulation. The AFM is introduced in the instrumental chain with all its complex scenes.

The user can observe the real phenomenon of approach-retract and feel it in terms of action-reaction in the hand. Once the AFM is linked to the real-time modelling station, the experimentalist does up-anddown movement with the key of the force feedback device by driving the piezoelectric tube and so, the AFM tip movement. The student is emerged in the nano-objects interaction by the visual rendering and by feeling the nanoscene dynamics through the force feedback device. Students do such experience during the practical works with stiff contact cantilever and silicon samples.

As a third step of the experimental procedure, the students disconnect the AFM from the platform and experienced other similar interactions by working on the virtual nano-scene model parameters. Changing the cantilever stiffness, the surface elasticity or the interaction thresholds they acquire reach information through real feelings on different interactions and their effects, like what is happening if a soft cantilever is used to explore a soft or a very rigid surface. This is a learning stage furnishing information otherwise impossible to obtain in a practical work without haptic feedback. It saves not only money because it avoids various samples and cantilevers buying, but also time since the material exchange in AFM device otherwise meticulous is not anymore necessary. This step avoids the trials and errors experiences, increasing the number of successful possible interaction to be tested.

After a practical work of three hours, students have to write a report where they describe and explain the phenomenon of the force curve. A first group of students (20 persons) studied this phenomenon using a classical commercial AFM with no haptic interface while a second group (20 persons) studied it using multisensorial interface.

The students who have used the multisensorial platform have described more efficiently and with more details the approach-retract phenomenon proving that they had a better understanding of this physical phenomenon.

The major differences observed between the two groups were: (1) in the understanding of the influence of cantilever spring constant value on the snap-on force intensity and on the snap-off distance value, (2) in the explanation of the hysteresis origin present in a force curve.

These differences could be explained by two points:

(1) The presence of the virtual nano-scene facilitate the second group to change the cantilever spring constant in a large interval and therefore to observe this parameter influence by visual and auditory rendering but also to feel directly in their hand the induced variations.

(2) They could generate interactions behaviours, which are not possible in a real manipulation by choosing extreme spring constant values. As example, by increasing the spring constant value, the hysteresis area in the approach-retract interaction can be reduced and even eliminated.

In comparison, the experiments realized by the first group on the classical AFM did not allow students to use a cantilever with such a high value of spring constant because it is technologically impossible to fabricate it.

The student's report analysis underlines the utility and the efficiency of adding haptic interface to a classical AFM in the learning process improvements.

\subsection{Interaction with Drosophilae leg through force feedback device}

During an educational project on MEMS - Micro Electro Mechanical System [14], master students has to find a way to measure and characterize Drosophilae leg reaction to an external stimulation. Drosophilae leg can be considered as biological microsystem very close to MEMS, as its diameter and size is around $100 \mu \mathrm{m}$ and it can react to a mechanical or electrical simulation. The idea was to use a specific AFM probe to interact with the end of the fly leg and to adapt and control in real time the foot-tip interaction (Fig. 6).

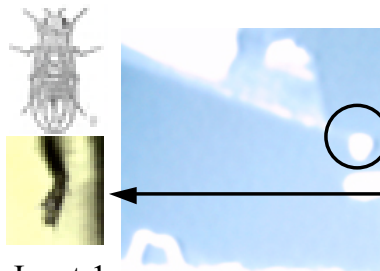

Inset 1: Drosophilae leg
Fig. 6 Setup for interacting with Drosophilae foot

To succeed in this goal, two main challenges had to be reached: (1) Fabricate a specific AFM probe which present a tip area on the same order as the drosophilae foot in order to apply a homogenous force on all the foot surface, (2) adapt and control tip-foot interaction according to the leg reaction in real time in order to avoid partial or total destruction of the foot or leg during the experience.

The specific probe was elaborated from a classical AFM cantilever $-300 \mu \mathrm{m}$ length, $35 \mu \mathrm{m}$ width, $1.5 \mu \mathrm{m}$ thickness- without tip at its end where a $50 \mu \mathrm{m}$ diameter latex sphere has been glued (Fig. 6).

The use of a modified AFM probe (Inset 2) was required for two reasons: (1) the surface interaction had to take place with all the surface of the foot, (2) the 
spring constant of the probe has to be small $(\sim 0.01 \mathrm{~N} / \mathrm{m}$ versus $1 \mathrm{~N} / \mathrm{m}$ for classical spring constant of contact cantilever) to minimize the risk of exerting a strong force intensity which could lead to a total or partial destruction of the Drosophilae leg/foot.

The second challenge was to adapt in real time the intensity of the applied force on the foot in function of the leg behaviour. The AFM is usually used to image and characterize samples, which cannot be deformed in vertical direction on such long distances as a leg. Indeed the fly leg can retract or stretch by itself on long distance -from few hundred of nanometers to few micrometers- according to the intensity of the pressure exerted on the foot.

A first attempt has been done using an AFM with a classical interface, which impedes fixing several parameters as the AFM probe velocity, the starting and stop points before realizing a force curve. Once these parameters are fixed, the force curve is done and the result display on the screen. Due to this lack of real time feedback and control, several force curves should have been needed to determine all the reaction panel of the leg to several kind of mechanical stimulation. These several force curves experiments are time consuming since they cannot be implemented automatically. They require an analysis of the previous recorded force curves to change the relevant parameters. Moreover, it is not a safe procedure because an error of curve interpretation can lead to a choice of not welladapted parameters inducing thus an alteration of the leg capability. Therefore, an AFM with a classical interface is not the adequate tool to realize this study.

In comparison, using the force feedback transducer allowed recording continuously the force variation versus time for different types of mechanical stimulation and minimizing the risk of leg alteration, as it is shown in Fig. 7.

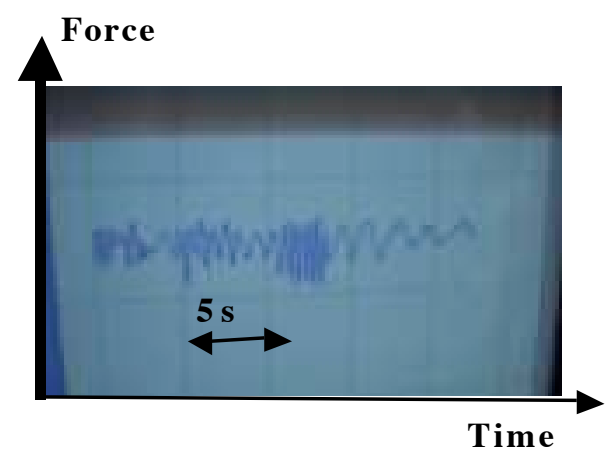

Fig. 7 Temporal variation of tip-foot force for different speed manipulations

The use of the force feedback system has improved the time of understanding the leg behaviour. Two main reasons sustain this: (1) an improvement of the experience time for recording force variation in accordance with different types of stimulation; (2) the haptic rendering of the contact interaction which could be instantly compared to the one obtained on a classical hard surface as silicon surface. Thanks to these two key points, it has been revealed straightforward and without analyzing force curves in detail that the leg presents an elastic behaviour for all the tested types of mechanical stimulation.

The user controlling the tip-foot interaction by adapting in real-time his hand impedance is illustrated in Fig. 8.

Moreover, this experience underlines the fact that the force feedback device linked to the AFM probe constitutes an adapted interface for the human hand to reach the sub-micrometer scale. This experience opens the door to the real time interaction with living insect through a nano-hand, where human could use their feeling, knowledge and own previous experiences to discover and act on this extraordinary world.

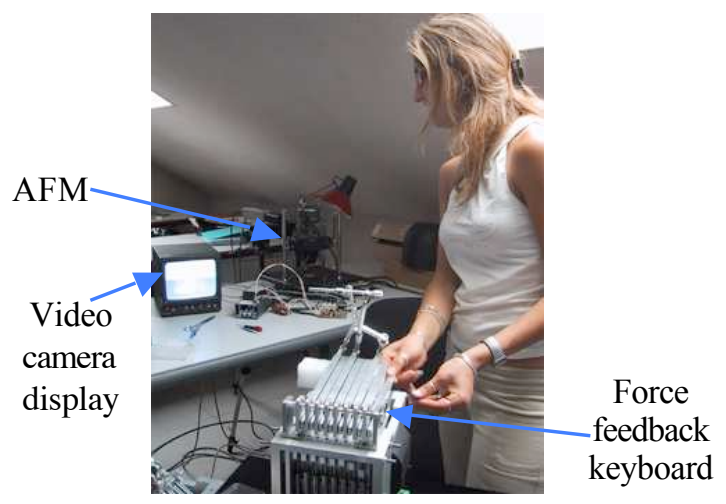

\section{Fig. 9 Student interacting with the Drosophilae leg}

\section{Conclusions}

The works presented in this paper show two main improvements in the learning process and in practical skills acquired by students.

(1) In comparison with a classical AFM interface, using a multisensorial platform equipped with a physically-based modelling engine, which works in real-time, improves the understanding of a physical complex phenomenon of approach-retract nanopalpating effect represented by a non-linear hysteretic force curve.

As the student handles directly the nanoscene, a change in the physical interaction is transferred in a variation perceived by human sensorial channels. Such perception is not possible with usual AFM interface that oblige students to make an effort of interpretation from symbolic graphic representation to sensed information. 
The benefits of using this platform as a new method of teaching are illustrated through the use of the physically-based model that reveals to be an appropriate tool to explain the role of each parameter involved in the studied interaction.

(2) Thanks to this platform, students were able to interact with non-conventional samples (Drosophilae leg) and to feel their rheological properties (elasticity, viscosity, plasticity, etc.), this type of manipulation being not successfully performed with a classical AFM. This experience opens a way to observe and characterise reactive samples at nanometer scale.

The work presented in this paper is the initial step in using non-traditional methods for education in nanophysics. In the future, this educational method will be applied to illustrate other complex physical and chemical interactions at the nanometer scale, as electrostatic and magnetic effects.

\section{Acknowledgements}

This work has been supported by the French National Center of Research (CNRS), the French Ministry of Culture and by the FP6 Network of Excellence IST-2002-002114 - Enactive Interfaces

\section{References}

[1] J. Israelachvili, "Molecular and surface forces", $2^{\text {nd }}$ edition, Academic Press, New-York, 1991

[2] G. Sankaranarayanan, S. Weghorst, M. Sanner, A. Gillet, A. Olson: Role of haptics in teaching structural molecular biology, Proc. of the $11^{\text {th }}$ Symposium on Haptics Interfaces for Virtual Environments and Teleoperator Systems, Los Angeles, CA(2003), 363-366

[3] J. Trindade, C. Fiolhais, V. Gil, J.C. Teixera: Virtual Environment of water molecules for learning and teaching science. Proc. of the Eurographics Workshop, GVE'99-Computer Graphics and Visualization Education'99, Coimbra, Portugal, 1999, 153-157

[4] C. Sauer, W.A. Hastings, A.M. Okamura: Virtual Environment for Exploring Atomic Bonding. Proc. of EuroHaptics 2004, Munich, Germany, June 5-7, 2004, 232-239
[5] W.L. Murphy, G.C. Spalding: Range of interactionsAn experiment in atomic and magnetic force microscopy. Am. J. Phys. 67 (10), October 1999, 905908

[6] J. Ringlein, M.O. Robbins: Understanding and illustrating the atomic origins of friction. Am. J. Phys. 72 (7), July 2004, 884-891

[7] J.J. Batter, P. Frederick, F.P. Brooks, Jr., "GROPE-I: A computer display to the sense of feel," Information Processing, Proc. IFIP Congress 1971, pp. 759-763.

[8] R.M. Taylor, W. Robinett, V. L. Chi, F. P. Brooks Jr., W. V. Wright, R. S. Williams, and E. J. Snyder: The Nanomanipulator: A Virtual-Reality Interface for a Scanning Tunneling Microscope. Computer Graphics: Proc. SIGGRAPH '93, Anaheim, CA, August, 127-134.

[9] G. Li, N. Xi, M. Yu, W.K. Fung: Development of augmented reality system for AFM-based nanomanipulation. IEEE/ASME Trans. on Mechatron., vol. 9, no.2, pp. 358-365, June 2004

[10] B. Cappella, G. Dietler: Force-distance curves by atomic force microscopy. ELSEVIER Press, Surface Science Reports 34 (1999) 1-104

[11] S. Marliere, D. Urma, J.L. Florens, F. Marchi: Multisenosrial interaction with a nano-scale phenomenon: the force curve. Proc. of EuroHaptics 2004, Munich, Germany, June 5-7, pp. 246-252

[12] J.L. Florens: Real time Bowed String Synthesis with Force Feedback Gesture. Invited Paper. Proceedings of Forum Acousticum. Sevilla. November 2002

[13] C. Cadoz, A. Luciani, J.L. Florens,"CORDIS-ANIMA:a Modelling and Simulation System for Sound and Image Synthesis: The General Formalism", CMJ vol.17/1-MIT Press, 1993

[14] D. Collard, H. Fujita, H. Toshiyoshi, B. Legrand, L. Buchaillot: Surface Micro-Machining - An Overview -Microsystem technology, Test and Reliability. MIGAS'02, Editor J. Boussey, Hermes Lavoisier, Vol. 2, pp. 35-49, 2002 